\title{
PARÁMETROS GENÉTICOS EN RASGOS DE LA PRODUCCIÓN LECHERA Y LA LONGEVIDAD DE VACAS MAMBÍ DE CUBA
}

\author{
GENETIC PARAMETERS IN MILK PRODUCTION AND LONGEVITY TRAITS \\ OF MAMBÍ DE CUBA COWS
}

\author{
Hernández, A. ${ }^{1}$, Ponce de León, R. ${ }^{1}$, García, S.M. ${ }^{2}$, García, R. ${ }^{2}$, Mora, M. ${ }^{1}$, Gutiérrez, M. ${ }^{1}$ \\ y Guzmán, G. ${ }^{1}$
}

\begin{abstract}
${ }^{1}$ Departamento de Genética. Instituto de Ciencia Animal. Apartado Postal 24. San José de Las Lajas. La Habana. Cuba. arelishdez@ica.co.cu

${ }^{2}$ Empresa Pecuaria Genética de Matanzas. Finca San Andrés. Limonar. Matanzas. Cuba. epgmmtz@atenas.inf.cu
\end{abstract}

\section{Palabras claves adicionales}

Correlaciones genéticas. Trópico.

\section{RESUMEN}

Se utilizaron los datos de 6335 vacas de la raza Mambí de Cuba (3/4 Holstein 1/4 Cebú) provenientes de 4 empresas genéticas, con partos desde 1980 a 2005, para estimar las heredabilidades $\left(\mathrm{h}^{2}\right)$ y las correlaciones genéticas y fenotípicas para la producción de leche en la primera lactancia (LPL), la producción de leche total acumulada por vida (LTV), vida productiva (VP) y habilidad de permanencia hasta 36 meses de vida productiva (HP36). Para estimar los componentes de varianza se utilizó el programa MTDFREML empleando un modelo animal uni o bivariado que incluyó como efecto fijo la combinación rebaño-año-época de primer parto, la edad al primer parto como covariable lineal y cuadrática y como efectos aleatorios el animal y el error. Los promedios para LPL, LTV, VP y HP36 fueron de $1990,9 \mathrm{~kg}, 9749,5 \mathrm{~kg}, 69,7$ meses, $0,77 \%$ y las heredabilidades fueron de $0,15 \pm 0,03,0,10 \pm 0,03$, $0,05 \pm 0,02,0,008 \pm 0,013$. Las correlaciones genéticas de la leche acumulada por vida con los rasgos de longevidad fueron altas $(0,59$ y 0,80$)$ y aquellas entre la producción lechera en la primera lactancia y los rasgos de longevidad fueron bajas y antagónicas $(-0,13$ y $-0,16)$. En el programa de mejoramiento genético de la raza, al seleccionar los futuros sementales, además de la producción

\author{
AdDITIONAL KEYWORDS \\ Genetic correlation. Tropic.
}

lechera se deben incluir rasgos que garanticen la permanencia de los animales en el rebaño.

\section{SUMMARY}

Data from 6335 cows of the Mambí de Cuba (3/4 Holstein 1/4 Zebu) breed from four genetic enterprises, with calving in the years from 1980 to 2005 were used to estimate the heritabilities and the genetic and phenotypic correlations of milk production in the first lactation (LPL), the total milk production accumulated for life (LTV), productive life (VP) and stayability for 36 month of productive life (HP36). The MTDFREML program was used to estimate the variance component using uni or bivariate animal model that included the fixed effect of herd-years-season of first calving, the age to first calving as lineal and quadratic covariable, and as random effect the animal and the residual. The means for LPL, LTV, VP and P36 were of $1990.9 \mathrm{~kg}, 9749.5 \mathrm{~kg}, 69.7$ month, $0.77 \%$ and the heritabilities were of $0.15 \pm 0.03,0.10 \pm 0.03$, $0.05 \pm 0.02,0.008 \pm 0.013$. The genetic correlations of milk production acumulated for life with the longevity traits were high (0.59 and 0.80$)$ and those between milk production in the first lactation and the longevity traits were low and antagonic 
(-0.13 and -0.16). In the genetic improvement of breed for selecting the future sire, besides milk production, traits that guarantee the stayability of the animals in the herd should be included.

\section{INTRODUCCIÓN}

La longevidad en el ganado lechero es una medida de su capacidad para sobrevivir en el rebaño ya sea al desecho voluntario (debido a baja producción de leche) como al involuntario (debido a problemas reproductivos o de salud).

Para estudiar la longevidad del ganado lechero se han utilizado diferentes variables siendo las más utilizadas (Hudson and Van Vleck, 1981; Short and Lawlor, 1992; Cruickshank et al., 2002; Ponce de León et al., 2002; Valencia et al., 2004; Chirinos et al., 2005; Daliri et al., 2008) la duración de la vida productiva en el hato (medida como la diferencia entre la fecha de primer parto y la fecha de desecho o del último registro de producción) o la vida productiva funcional cuando se corrige por la producción lechera, el número total de lactancias, la producción de leche acumulada en todas las lactancias o la producción de leche acumulada a la tercera lactancia y las habilidades de permanencia hasta cierto número de meses de edad (36, 48, 60, 72, 84 meses) o cierto número de meses después del primer parto (12, 24, 36 meses).

La metodología de máxima verosimilitud restringida con el uso de un modelo animal ha sido utilizada para estimar parámetros genéticos en características de longevidad (Short and Lawlor, 1992; Cruickshank et al., 2002; Valencia et al., 2004; Daliri et al., 2008) y recientemente han comenzado a usarse técnicas de análisis de supervivencia (Chirinos et al., 2005).

Actualmente, hay una tendencia mundial al uso de múltiples características en los programas de mejoramiento genético de los bovinos lecheros donde además de las características de la producción lechera se han incluido características de longevidad, tipo y aquellas relacionadas con la salud del animal, mediante el empleo de índices de selección, para lo cual es necesario estimar las heredabilidades y correlaciones genéticas y fenotípicas entre las características que serán incluidas en el índice (VanRaden y Klaaskate, 1993; Jairath et al., 1995).

Estudios previos en el Holstein (Ponce de León y Guzmán, 1993) y en el Siboney de Cuba (5/8 Holstein 3/8 Cebú) en nuestras condiciones ambientales (Ponce de León et al., 2002; Suárez et al., 2005) han analizado la relación entre la producción lechera y las características de longevidad. Sin embargo, en la raza Mambí de Cuba (3/4 Holstein 1/4 Cebú) no hay referencias al respecto. Por ello resulta de interés estimar las heredabilidades, las correlaciones genéticas y fenotípicas de la producción lechera, y en particular de la producción en primera lactancia con los rasgos de longevidad. Esta información podría ser útil para la realización de futuras estrategias genéticas en el programa de mejoramiento genético del ganado Mambí de Cuba.

\section{MATERIALESY MÉTODOS}

Para estudiar los parámetros genéticos de los rasgos de producción lechera en primera lactancia y por vida, así como sus relaciones con rasgos de longevidad se tomó la información de vacas Mambí de Cuba (3/4 Holstein, 1/4 Cebú) que causaron baja, pertenecientes a cuatro ganaderías (empresa Pecuaria Genética de Matanzas, empresa Bacuranao, empresa Los Naranjos y empresa Camilo Cienfuegos). Todas estaban localizadas en la región occidental de la República de Cuba, situada en el golfo de México, entre 20 a $23^{\circ} \mathrm{N}$ y 74 a $85^{\circ} \mathrm{O}$. Según Anonimo (2009) en la mayor parte de Cuba el clima predominante es de tipo cálido tropical con dos estaciones: la lluviosa, comprendida entre los meses de mayo a octubre, y la seca de noviembre a abril. La temperatura media anual es de $24^{\circ} \mathrm{C}$, la humedad relativa media presenta promedios cercanos al 80\% y las precipitaciones superan los 


\section{PARÁMETROS GENÉTICOS EN PRODUCCIÓN Y LONGEVIDAD DE VACAS MAMBÍ}

$3000 \mathrm{~mm}$ al año.

El sistema de alimentación fue basado en pastoreo. Las vacas lactantes consumieron fundamentalmente pasto estrella (Cynodon nlemfuensis), pasto pangola (Digitaria decumbens) y guinea (Panicum maximum), además recibieron diariamente concentrado comercial de forma individual a razón de $1 \mathrm{~kg}$ de concentrado para 2,5 kg de leche cuando las producciones individuales excedían los 2 kg/día. Durante el período de seca fue suministrado suplementación de caña de azúcar (Saccharum officinarum) y king grass (Penisetum purpureum) en forma de forraje, úrea y sales minerales. Las hembras no lactantes se mantuvieron en las mismas condiciones de pastoreo. A los 30 días pre-parto recibieron suplementación con concentrado a razón de $2 \mathrm{~kg}$ /día. Se realizó ordeño mecánico 2 veces al día.

Como rasgos de la producción lechera se estudiaron la producción LPL y la producción LTV y como rasgos de longevidad la VP determinada como los meses desde el primer parto a la baja y la habilidad de permanencia hasta los HP36 asignándose 0 cuando la vaca no llegó a los 36 meses de vida productiva y 1 si su vida productiva fue igual o mayor a 36 meses.

Se utilizaron como punto de partida 50 420 lactancias de la producción lechera acumulada hasta los 305 días. Para garantizar una mayor precisión en la estimación de los parámetros se eliminaron aquellas lactancias con menos de 100 días (5,36\% de los datos), las edades al parto inferiores a los 24 meses $(0,06 \%)$ y las producciones con menos de 300 kg (4,23\%). También se eliminaron los valores por encima y por debajo de 3 desviaciones estándar de la media y los grupos de contemporáneos conformados por menos de 3 animales para un total de 42548 observaciones.

A partir del fichero depurado se realizó el cálculo de la producción LTV sumando la producción de leche acumulada hasta los 305 días de todas las lactancias registradas de cada vaca. Este nuevo fichero de produc- ciones totales contó con 20683 observaciones.

En el archivo final de datos se utilizaron todas las vacas que tuvieron información para las cuatro variables, haciendo un total de 6335 vacas con años de primer parto entre 1980 y 2005. El fichero de pedigrí quedó conformado por 5549 madres y 213 padres.

Para estimar los componentes de varianza y parámetros genéticos se empleó el programa MTDFREML (Boldman et al., 1995). Se utilizó un modelo animal univariado para estimar las heredabilidades de cada una de los rasgos estudiados. El modelo utilizado en notación matricial fue:

$$
y=X b+Z a+e
$$

donde:

$y$ : vector $(n \times 1)$ de observaciones;

$X$ : matriz de incidencia ( $\mathrm{nxp}$ ) de efectos fijos, donde se incluyó el efecto del grupo de contemporáneo (rebaño-año-época de primer parto) y la edad al primer parto como covariable lineal y cuadrática;

b: vector ( $p \times 1)$ de efectos fijos desconocido;

$Z$ : matriz incidencia ( $\mathrm{n} \times q)$ de efectos aleatorios, que incluyó el animal;

a: vector ( $q \times 1)$ de efectos genéticos aditivos;

e: vector (nx1) aleatorio (error). Al efectuar los análisis, se supuso que los elementos de a y e se distribuyeron normalmente, que no estaban correlacionados y que los elementos de $y$ se distribuyeron normalmente.

Las correlaciones genéticas y fenotípicas entre los rasgos se obtuvieron mediante análisis bivariados empleando los mismos efectos fijos y aleatorios incluidos en los análisis univariados.

\section{RESULTADOSYDISCUSIÓN}

La descripción estadística de las características de la producción lechera y la longevidad se presentan en la tabla I donde se aprecia una mayor variabilidad para la producción lechera acumulada por vida y la 
vida productiva, con coeficientes de variación de 66,2\% y 55\%, respectivamente.

La producción lechera en primera lactancia y la producción lechera por vida fueron inferiores a los $2243 \pm 12 \mathrm{~kg}$ y $8135 \pm 72 \mathrm{~kg}$ obtenidos en el Siboney de Cuba bajo las condiciones de la empresa Nazareno (Ribas et al., 2001).

El promedio para la vida productiva fue superior a lo observado en el Holstein bajo nuestras condiciones ambientales con 45,6×0,8 (Ponce de León y Guzmán, 1991), en el Siboney de Cuba (Ribas et al., 2001) con $51,9 \pm 0,5$ meses y a lo encontrado en el Holstein en Estados Unidos por Tsuruta et al. (2005) con 34,3 $\pm 0,8$ meses. Los rebaños altos productores tienen menores promedios de vida productiva principalmente por un incremento del reemplazo voluntario (Van Arendonk, 1985).

La habilidad de permanencia hasta 36 meses de vida productiva superó los $0,68 \pm 0,01 \%$ y los $0,59 \pm 0,01 \%$ encontrados en el Siboney de Cuba por Ribas et al., 2001 y Ponce de León et al., 2002.

La varianza genética aditiva $\left(\sigma^{2} a\right)$, varianza residual $\left(\sigma^{2} \mathrm{e}\right)$, varianza fenotípica $\left(\sigma^{2} f\right)$ y heredabilidades de los rasgos de longevidad y la producción lechera se muestran en la tabla II y las correlaciones fenotípicas y genéticas en la tabla III.

La heredabilidad para la producción lechera en primera lactancia coincide con la obtenida en el Siboney de Cuba (Gonzáles et al., 2005) y en el Holstein en Cuba (Palacios-Espinosa et al., 2007). Los estimados para la producción lechera por vida fueron similares a los de Ribas et al. (2001) en el Siboney de Cuba perteneciente a la empresa Nazareno y aquellos para la habilidad de permanencia hasta 36 meses de vida productiva, fueron bajos al igual que los obtenidos en el trabajo citado anteriormente.

La heredabilidad de la vida productiva coincide con los estimados en vacas Holstein por Ponce de León y Guzmán (1991) con 0,08 , con los 0,07 para la vida productiva verdadera por Short y Lawlor (1992), los
Tabla I. Media, desviación estándar (DE) y coeficiente de variación (CV) de los rasgos de la producción lechera y la longevidad de vacas Mambí de Cuba. (Mean, standard deviation (DE) and coefficient of variation (CV) for milk production and longevity traits of Mambí de Cuba cows).

\begin{tabular}{lccc}
\hline Rasgos & Media & DE & CV (\%) \\
\hline EPP (meses) & 38,9 & 3,8 & 10,5 \\
LPL (kg) & 1990,9 & 813,9 & 40,9 \\
LTV ( kg) & 6610,5 & 2273,0 & 66,2 \\
VP (meses) & 69,7 & 38,4 & 55,0 \\
HP36 (\%) & 0,77 & 0,42 & 54,7
\end{tabular}

EPP: edad al primer parto; LPL: producción lechera en primera lactancia; LTV: producción lechera por vida; VP: vida productiva; HP36: habilidad de permanencia hasta 36 meses de vida productiva.

0,08 para la vida productiva funcional por Jairath et al. (1998) y los 0,04 y 0,03 para la vida productiva verdadera y funcional, respectivamente (Daliri et al., 2008). También se corresponde con los 0,06 y 0,04 para la vida productiva verdadera y funcional en el ganado Pardo Suizo en Italia (Cassandro et al., 1999) y con los 0,054 para la vida productiva en el Siboney de Cuba (Ponce de León et al., 2002 y Suárez et al., 2005).

La producción lechera en primera lactancia presentó correlaciones fenotípicas bajas $(0,01$ a 0,05$)$ con otros rasgos como la producción lechera por vida, la vida productiva y la habilidad de permanencia hasta 36 meses de vida productiva. Aliji et al. (2007) en el Holstein, en Túnez, obtuvieron resultados similares.

Las correlaciones genéticas entre la leche acumulada por vida y los rasgos de longevidad fueron altas $(0,59$ y 0,80$)$. Sin embargo, los estimados entre la leche producida en primera lactancia y los rasgos de longevidad fueron negativos y bajos (-0,13 y $-0,16)$ y aquellos entre la leche producida en primera lactancia y la leche acumulada 


\section{PARÁMETROS GENÉTICOS EN PRODUCCIÓN Y LONGEVIDAD DE VACAS MAMBÍ}

Tabla II. Estimadores de la varianza genética aditiva $\left(\sigma^{2} a\right)$, varianza residual $\left(\sigma^{2} e\right)$, varianza fenotípica $\left(\sigma^{2} f\right)$ y heredabilidad $\left(h^{2}\right)$ en rasgos de la producción lechera y la longevidad de vacas Mambí de Cuba. (Estimators of additive genetic variance $\left(\sigma^{2} a\right)$, residual variance $\left(\sigma^{2} e\right)$, phenotypic variance $\left(\sigma^{2} \mathrm{f}\right)$ and heredability $\left(\mathrm{h}^{2}\right)$ in milk production and longevity traits of Mambí de Cuba cows).

\begin{tabular}{lcccr}
\hline Rasgos & LPL & LTV & VP & HP36 \\
\hline$\sigma^{2} \mathrm{a}$ & 24452 & 1706070 & 42,50 & 63571 \\
$\sigma^{2} \mathrm{e}$ & 137923 & 14548900 & 822.70 & 7604130 \\
$\sigma^{2} \mathrm{f}$ & 162375 & 16254970 & 865.20 & 7667701 \\
$\mathrm{~h}^{2}$ & $0,15 \pm 0,03$ & $0,10 \pm 0,03$ & $0,05 \pm 0,02$ & $0,008 \pm 0,013$
\end{tabular}

LPL: producción lechera en primera lactancia; LTV: producción lechera por vida; VP: vida productiva; HP36: habilidad de permanencia hasta los 36 meses de vida productiva.

por vida fue sólo de 0,07 .

Estos resultados indican que las vacas con mayores producciones en su primer parto no fueron las más longevas, debido a que no hubo un tratamiento diferencial para las vacas altas productoras. Las mismas están desfavorecidas en la alimentación pues la cantidad de concentrado que se les ofrece está en correspondencia con la media del grupo de alta, que es inferior a su promedio de producción lechera, y por consiguiente no cubren sus requerimientos nutricionales. Esto provoca que las vacas altas productoras presenten problemas reproductivos (no presentan celo y no que- dan gestantes) por lo que son las más propensas a causar baja.

Los resultados obtenidos no se corresponden con los estudios realizados en las regiones templadas y con ganadería desarrollada. Entre ellos podemos mencionar los resultados de Hudson y Van Vleck (1981) en el Holstein, en Estados Unidos, donde las correlaciones genéticas entre la producción lechera en primera lactancia y la permanencia hasta 36, 48, 60, 72 y 84 meses de edad presentaron valores entre 0,47 y 0,65. De Lorenzo y Everett (1982) para la misma raza obtuvieron estimados de 0,34 y 0,47 entre la producción lechera en primera lac-

Tabla III. Correlaciones genéticas (arriba de la diagonal) y fenotípicas (debajo de la diagonal) en rasgos de la producción lechera y la longevidad de vacas Mambí de Cuba. (Genetic (above the diagonal) and phenotypic (below the diagonal) correlations in milk production and longevity traits of Mambí de Cuba cows).

\begin{tabular}{lcccc}
\hline & LPL & LTV & VP & HP36 \\
\hline LPL & - & $0,07 \pm 0,23$ & $-0,6 \pm 0,21$ & $-0,13 \pm 0,49$ \\
LTV & $0,01 \pm 0,03$ & - & $0,59 \pm 0,04$ & $0,80 \pm 0,30$ \\
VP & $0,04 \pm 0,03$ & $0,59 \pm 0,02$ & - & $0,88 \pm 0,41$ \\
HP36 & $0,05 \pm 0,04$ & $0,45 \pm 0,02$ & $0,86 \pm 0,01$ & - \\
\hline
\end{tabular}

LPL: producción lechera en primera lactancia; LTV: producción lechera por vida; VP: vida productiva; HP36: habilidad de permanencia hasta 36 meses de vida productiva. 
tancia y la permanencia hasta 48 y 72 meses, respectivamente. Cassandro et al. (1999) en el ganado Pardo Suizo, en Italia, obtuvieron una correlación genética de 0,66 entre la producción de leche en primera lactancia y la vida productiva verdadera (tiempo del primer parto hasta la eliminación, excluyendo el período seco). En Holstein, en México, Valencia et al. (2004) apreciaron que la producción de leche en la primera lactancia presentó correlaciones genéticas medias con los rasgos de longevidad $(0,33-0,38)$ y altas con la producción de leche acumulada en la tercera lactancia $(0,64)$.

En los estudios previos realizados en nuestro país en el Holstein (Ponce de León y Guzmán, 1993) y en el Siboney de Cuba (Ponce de León et al., 2002) se encontraron valores más antagónicos en las correlaciones genéticas entre la leche en primera lactancia y la longevidad (-0,60 a $-0,64$ y de $-0,30$ a $-0,61$, respectivamente) que los obtenidos en el presente estudio.

El mecanismo biológico relacionado con la selección por incremento de la producción lechera y el decline en la adaptabilidad y el bienestar de las vacas lecheras fue descrito por Ingvartsen et al. (2003) quiénes plantearon que las vacas altas productoras de leche necesitaban movilizar las reservas corporales para mantener su producción y en el primer tercio del periodo de lactancia, aún cuando la energía consumida superara sus requerimientos, entraban en un estado de balance energético negativo perdiendo excesivamente su condición corporal. Los animales con alto potencial genético requieren una mayor demanda de energía, lo cual provoca un incremento del metabolismo bajo, de la movilización de los tejidos y del estrés metabólico. Este último es el causante de una disminución en el sistema reproductivo, en el sistema inmune y en la capacidad buferante; los cuales a su vez, causan una disminución en el bienestar de la vaca.

Oltenacu y Algers (2005) ofrecen una explicación de lo que ocurre en países tro- picales planteando que el empleo de prácticas de manejo inadecuadas puede incrementar el estrés metabólico, lo cual es de mayor repercusión, en sistemas de producción de leche sostenibles, que maximizan el uso de forrajes.

En los trópicos, otros autores como Giuliani et al. (1997) al estudiar la longevidad de la raza Carora (5/8 pardo suizo x 3/8 criollo) observaron una fuerte incidencia del manejo en la permanencia de los animales en el rebaño. Las fincas con alto y mediano nivel tecnológico (por lo tanto más productivas) presentaron valores de sobrevivencia menores a los detectados en fincas de bajo nivel tecnológico, debido a una mayor presión de selección para mejorar la producción de leche y consecuentemente una fuerte eliminación de los animales menos productivos.

Johnson (1991) planteó que es importante profundizar el estudio de si en el aumento del número de eliminaciones, concurren también factores debidos al stress a que se someten los animales que presentan buenas producciones en un medio bastante difícil como el tropical.

En los últimos años hay una tendencia mundial a incluir en los índices de selección características de durabilidad, salud y reproducción. Dentro del componente de durabilidad se consideraron varios rasgos como la longevidad, el tamaño del cuerpo, la ubre completa, piernas y patas, la puntuación final, el temperamento lechero y otros. Miglior et al. (2005) en una revisión realizada en varios países encontraron que el índice TOP, de Gran Bretaña empleaba el mayor énfasis relativo en la durabilidad (42\%), seguido del TPI en Estados Unidos (41\%), el RZG en Alemania (40\%), el LPI en Canadá (38\%), el ICO en España (38\%), el APR en Australia (16,5\%) y el PLI en Gran Bretaña (20\%). Los índices con un mayor énfasis en la longevidad fueron el DPS en Holanda, con un 26\%, seguido del RZG en Alemania (25\%) y el EBI en Irlanda (23\%).

Short y Lawlor (1992) y Harris et al. 


\section{PARÁMETROS GENÉTICOS EN PRODUCCIÓN Y LONGEVIDAD DE VACAS MAMBÍ}

(1993) plantearon que la mejora genética de la vida en el rebaño (o longevidad) usando su evaluación directa era difícil porque este rasgo presentaba baja heredabilidad, era medido sólo en vacas y no tenía repetibilidad, además de no poder ser medido en etapas tempranas de la vida (Sewalem et al., 2004). Cruickshank et al. (2002) y Neuenschwander et al. (2005) argumentaron que la evaluación indirecta de la vida en el rebaño podía realizarse a través de características correlacionadas genéticamente como los rasgos del tipo.

Actualmente en Cuba como criterio de

\section{BIBLIOGRAFÍA}

Anonimo. 2009. El clima en Cuba. www.bedincuba. com/2climaencuba.htm. (07/02/09).

Ajili, N., Rekik, B., Ben Gara, A. and Bouraoui, R. 2007. Relationships among milk production, reproductive traits, and herd life for Tunisian Holstein-Friesian cows. Afri.J. Agr. Res., 2: 047-051.

Boldman, K. G., Kriese, L.A., Van Vleck, D., Van Tassell, C.P. and Kachman, S.D. 1995. A manual for use of MTDFREML. A set of programs to obtain estimates of variances and covariances (Draft). Agricultural Research Service. Washington DC. 114 pp.

Cassandro, M., Ghiroldi, S., Bagnato, A., Gallo, L., Carnier, P. and Santus, E. 1999. Genetic evaluation of longevity in Italian brown cattle breed. Proceeding International Workshop EU Concerted Action GIFT. pp. 73-81.

Chirinos, Z., Hernández, D. y Carabaño, M.J. 2005. Relación entre caracteres de tipo y longevidad en ganado frisón utilizando técnicas de análisis sobrevivencia. Rev. Cientifica, 15: 263-271.

Cruickshank, J., Weigel, K.A., Dentine, M.R. and Kirkpatrick, B.W. 2002. Indirect prediction of herd life in Guernsey dairy cattle. J. Dairy Sci., 85: 1307-1313.

Daliri, Z., Hafezian, H., Shad Parvar, A. and Rahimi, G. 2008. Genetic relationships among longevity, milk production and linear type traits in Iranian Holstein cattle. J. Anim. Vet. Adv., 7: 512-515. www.medwelljournals.com/fulltext/java/2008/ 512-515.pdf. (25/11/08). selección en los toros lecheros se utiliza solamente la producción lechera de sus hijas. La correlación genética negativa de la producción lechera en la primera lactancia con la vida productiva ha evidenciado la importancia de que en el programa de mejoramiento genético de la raza Mambí de Cuba, para la elección de los futuros sementales, se incluyan rasgos que garanticen la permanencia de los animales en el rebaño. Por ello resulta necesario realizar estudios futuros sobre la relación de la longevidad con otras características incluyendo las del tipo, como predictoras indirectas de la longevidad.

De Lorenzo, M.A. and Everet, R.W. 1982. Relationship between milk and fat production, type, and stayability in Holstein sire evaluation. J. Dairy Sci., 65: 1277-1285.

Giuliani, M.G., Rizzi, R., Hanh, M. and Cerutti, F. 1997. La longevidad en la raza Carora: efectos del nivel productivo de la finca y de los años. Arch. Latinoam. Prod. Anim., 5: 506-508. www.alpa. org.ve/PDF/Arch\%2005\%20Suplemento/ GM15.pdf. (18/02/09).

Gonzáles, D., Guerra, D., Evora, J.C., Ortiz, J. y Acosta, M. 2005. Parámetros genéticos de primera lactancia en la raza Siboney utilizando los polinomios de Legendre. III Congreso Internacional de Mejoramiento Animal. La Habana. Cuba.

Harris, B.L., Freeman, A.E. and Freeman, E. 1993. Economic weights for milk yield traits and herd life under various economic condition and production quotas. J. Dairy Sci., 75: 11471153.

Hudson, G.F.S. and Van Vleck, L.D. 1981. Relationship between production and stayability in Holstein cattle. J. Dairy Sci., 64: 2246-2250. Ingvartsen, K.L., Dewhurst, R.J. and Friggens, N.C. 2003. On the relation ship between lactational performance and health: is it yield or metabolic imbalance that causes diseases in dairy cattle? A position paper. Livest. Prod. Sci., 83: 277308.

Jairath, L.K., Hayes, J.F. and Cue, R.I. 1995. Correlations between first lactation and lifetime performance traits of Canadian Holsteins. J. 
Dairy Sci., 78: 438-448.

Jairath, L., Dekkers, J.C.M., Schaeffer, L.R., Liu, Z., Burnside, E.B. and Kolstad, B. 1998. Genetic evaluation for herd life in Canada. J. Dairy Sci., 81: 550-562.

Johnson, H.D. 1991. In: Feeding dairy cows in the Tropics. FAO. Roma. 86 pp.

Miglior, F., Muir, B.L. and Van Doormaal, B.J. 2005. Selection indices in Holstein cattle of various countries. J. Dairy Sci., 88: 1255-1263.

Neuenschwander, T., Kadarmideen, H.N., Wegmann, S., De Haas, Y. 2005. Genetics of parity-dependent production increase and its relationship with health, fertility longevity, and conformation in Swiss Holsteins. J. Dairy Sci., 88: 1540-1551.

Oltenacu, P. A. and Algers, B. 2005. Selection for increased production and the welfare of dairy cows: Are new breeding goals needed?. Ambio, 34: 311-315.

Ponce de León, R. y Guzmán, G. 1991. Heredabilidades y factores que afectan la longevidad y reproducción por vida en vacas Holstein. Rev. Cubana Cienc. Agríc., 25: 237243.

Palacios-Espinoza, A., Espinoza Villavicencio, J.L., González-Peña, D., Guerra Iglesias, D., De la Peña, R.L. and Rodríguez Almeida, F. 2007. Estimation of covariance components for the first four lactations in Holstein cattle according to different models. Zootec. Trop., 25: 9-18.

Ponce de León, R. y Guzmán, G. 1993. Relación entre la supervivencia de vacas y la producción en primera lactancia. 1. Correlaciones genéticas y entre valores genéticos. Rev. Cubana Cienc. Agríc., 27: 17-24.

Ponce de León, R., Ribas, M.L, Guzmán, G., Gutiérrez, M. y Mora, M. 2002. Longevidad, supervivencia y producción por vida de la nueva raza Siboney de Cuba. II Congreso Internacional de Mejoramiento Animal. La Habana.

Ribas, M., Ponce de León, R., Ajete, A., Mederos,
R.E., Gutiérrez, M. y Sosa, E. 2001. Mejoramiento genético de la producción de leche bovina bajo las condiciones actuales de producción. Informe final de proyecto. ICA. La Habana. Cuba. $71 \mathrm{pp}$.

SAS. 2002. User's guide statistics. SAS Inst. Cary. USA.

Sewalem, A., Kistemaker, G.J., Migliorand, F. and Van Doormaal, B.J. 2004. Analysis of the relationship between type traits and functional survival in Canadian Holsteins using a weibull proportional hazards model. J. Dairy Sci., 87: 3938-3946.

Short, T.H. and Lawlor, T.J. 1992. Genetic parameters of conformation traits, milk yield, and herd life in Holsteins. J. Dairy Sci., 75: 19871998.

Suárez, M.A., Fernández, L., Pérez, T., Quincosa, J., Álvarez, A., Pérez, E., Evora, J.C., Menéndez, A., González, M.T. y Marrero, A. 2005. Nuevo genotipo lechero para la producción de leche y carne. Informe final de proyecto. Siboney de Cuba. 198 pp.

Tsuruta, T., Misztal, I. and Lawlor, T.J. 2005. Changing definition of productive life in U.S. Holsteins: effect on genetic correlations. $J$. Dairy Sci., 88: 1156-1165.

Valencia, M., Ruíz, F.J. y Montaldo, H.H. 2004. Estimación de parámetros genéticos para características de longevidad y producción de leche en ganado Holstein en México. INCI., 29: 52-56. www.scielo.org.ve/scielo.php?script= sci_arttext\&pid=S0378184420040 00100013 \&lng=es\&nrm=iso. (04/05/07).

Van Arendonk, J.A.M. 1985. Studies on the replacement policies in dairy cattle. II. Optimum policy and influence of changes in production and prices. Livest. Prod. Sci., 13: 101-121.

VanRaden, P.M. and Klaaskate, E.J.H. 1993. Genetic evaluation of length of productive life including predicted longevity of live cows. J. Dairy Sci., 76: 2758-2764. 